\title{
Dictynna
}

Dictynna

Revue de poétique latine

$10 \mid 2013$

Varia

\section{La 'cuisine' de Virgile. À propos du centon virgilien De panificio}

Séverine Clément-Tarantino

\section{(2) OpenEdition}

Journals

Édition électronique

URL : http://journals.openedition.org/dictynna/1014

DOI : 10.4000/dictynna.1014

ISSN : 1765-3142

Référence électronique

Séverine Clément-Tarantino, "La 'cuisine' de Virgile. À propos du centon virgilien De panificio »,

Dictynna [En ligne], 10 | 2013, mis en ligne le 19 décembre 2013, consulté le 10 septembre 2020. URL : http://journals.openedition.org/dictynna/1014; DOI : https://doi.org/10.4000/dictynna.1014

Ce document a été généré automatiquement le 10 septembre 2020.

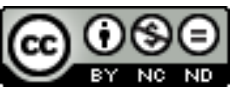

Les contenus des la revue Dictynna sont mis à disposition selon les termes de la Licence Creative Commons Attribution - Pas d'Utilisation Commerciale - Pas de Modification 4.0 International. 


\title{
La 'cuisine' de Virgile. À propos du centon virgilien De panificio
}

\author{
Séverine Clément-Tarantino
}

Virgile [arrivé à Rome, après avoir étudié la médecine et l'astrologie à Naples] était devenu le protégé du maître de l'écurie [d'Auguste]. Il sut

soulager des chevaux atteints d'affections variées; de plus, en les examinant, il sut dire qui

étaient les parents d'un certain nombre de chevaux; peu après, il fit la même chose pour des

chiens : cela poussa Auguste, par deux fois, à augmenter sa ration de pain. Auguste, qui doutait de l'identité de son père, espérant que Virgile la lui ferait connaitre, le consulta donc pour savoir de quel père il était né ; en demandant la permission, Virgile dit : «si j'osais te dire librement ce que je pense, je te dirais que tu es né

d'un père boulanger. » Cette réponse stupéfia

Auguste. Il poursuivit : « en effet, pour récompenser la finesse de mon jugement, tu as fait augmenter ma ration de pain : c'était bien là

le devoir d'un boulanger ou d'un fils de boulanger ! » Cette plaisanterie charme César, qui dit ensuite : «Tu ne recevras plus les dons d'un boulanger mais d'un roi magnanime ». Dès lors il devint chaque jour plus cher à Auguste... (Josse Bade, Préambule à l'édition commentée de Virgile ${ }^{1}$ )

1 Le but que je me fixe ici est une meilleure compréhension d'un petit texte tout à fait singulier, qui n'est pas de Virgile mais implique partout Virgile: le centon virgilien intitulé De Panificio, "Sur la fabrication du pain " ${ }^{2}$. Le centon, en tant que forme littéraire, connaît actuellement une revalorisation : plusieurs travaux récents tout à fait 
passionnants ont été consacrés en particulier aux centons de Virgile ${ }^{3}$, et il n'est plus question d'exclure ceux-ci des éditions de l'Anthologie latine, comme cela avait été malheureusement le cas pour la dernière édition de ce corpus pour Teubner ${ }^{4}$ !

Je ne rappellerai que quelques éléments de base ${ }^{5}$ : le centon est un texte formé de l'assemblage de segments d'un texte antérieur, généralement - dans sa forme la plus identifiable et, dans sa forme antique - d'un seul auteur. (Dans le cas des centons de Virgile, on peut trouver réunis des segments de vers ou des vers pris dans les Bucoliques, les Géorgiques et l'Énéide, l'important étant que seul Virgile soit ainsi directement cité.) Les centons obéissent à des contraintes précises, qui se sont surtout imposées dans la pratique même de cette forme; mais les centons virgiliens possèdent aussi une codification précise, qui a été fournie par le poète du IV ème siècle Ausone, dans l'épître dédicatoire dont il fait précéder son propre centon virgilien, un des plus célèbres en raison de l'écart maximal qu'il introduit entre Virgile et ce qu'il en fait : c'est le Centon nuptial, avec une section pornographique très fameuse, où Virgile est complètement décoiffé, ou plutôt "défloré» comme la jeune vierge (cf. le surnom de Virgile, Parthenias) lors de sa nuit de noces ${ }^{6}$.

Dans le cas du De Panificio également, l'écart entre le texte source et le texte d'accueil (le centon lui-même) peut apparaître comme très important : il s'agit en effet, à la base, d'utiliser des vers de Virgile - un des plus grands poètes de Rome et même, sans doute, le plus grand poète de Rome pour les auteurs de centons - pour parler d'un sujet très quotidien et très concret. Quoique Virgile ait aussi illustré un genre et surtout, de son propre aveu, un style et des thèmes poétiques humbles (dans les Bucoliques et les Géorgiques ${ }^{7}$ ), il est néanmoins généralement considéré comme un tout, et en tant que tout, comme un poète du grand style, voire comme un poète de la grandeur tout court. (Même aux époques où ce n'est pas l'Énéide qui est le poème virgilien le plus prisé, ses partisans ont à cœur de révéler la grandeur des deux autres poèmes - ou de l'un des deux -, malgré la petitesse que comportent parfois leurs sujets. $)^{8}$

Il me semble, en effet, que, même si on a (e.g.) les Bucoliques particulièrement en tête, quand on découvre le centon De Panificio, on ne peut pas ne pas être d'abord surpris par le décalage entre la langue utilisée dans ce court poème - le 'virgilien', si je puis dire et le sujet traité - la fabrication du pain. Ma question est alors la suivante : est-ce que ce décalage, avec tous les détournements qu'il implique (toutes les dé-contextualisations de vers que l'on peut imaginer, en admettant en outre, comme par principe, que l'on passe constamment d'un contexte d'origine élevé à un contexte d'arrivée bas) - est-ce que ce décalage signifie nécessairement que le centoniste a voulu composer une « parodie » de Virgile?

5 La définition de la parodie - concept qui peut être très fuyant - avec laquelle je vais travailler, est celle qu'a utilisée Scott Mc Gill en interprétant le centon de cette manière: comme un texte où la poésie 'haute' de Virgile est systématiquement détournée et tirée vers le bas, dans une intention qui est, sinon véritablement de railler Virgile - de manière critique - de faire rire, de produire un effet comique ${ }^{9}$. Or c'est une interprétation qui ne me convainc pas totalement, parce que je ne pense pas que les détournements que je viens d'évoquer soient aussi importants que l'on pourrait croire, ou que Scott Mc Gill le donne à penser. C'est ce que je me propose de vérifier en étudiant le texte du centon; mais, pour tâcher de comprendre encore mieux ce texte, je commencerai par le replacer dans la tradition poétique latine en considérant d'autres 
traitements poétiques de son sujet - la panification, le pain - ainsi que la place des thèmes de l'alimentation et de la cuisine dans l'œuvre de Virgile.

\section{Le centon et son sujet alimentaire}

6 Le centon De Panificio est un des deux centons virgiliens non chrétiens ${ }^{10}$ conservés dont le sujet est tiré du quotidien : l'autre pièce est le De alea (Sur le jeu de dés), poème plus long et plus obscur, avec lequel le De Panificio est traité dans le chapitre 3 de l'ouvrage que Scott Mc Gill a consacré aux centons virgiliens non chrétiens. Scott Mc Gill pense que les deux pièces ont été composées en Afrique (comme les autres textes poétiques du Codex Salmasianus, recueil réalisé Afrique au $6^{\text {ème }}$ siècle et inclus par la suite dans l' Anthologie latine) et il les situe toutes deux dans une fourchette large 203-53411.

Le De Panificio se présente comme relativement bref avec seulement onze vers ; il paraît être mutilé en son début, plutôt abrupt, avec un ipse qui isole la figure du 'chef' boulanger en l'opposant implicitement au reste du personnel, rendu présent (de manière plutôt abrupte également) par les pluriels des v. 9-10. La scène décrite paraît claire dans ses grandes lignes : un boulanger pétrit la pâte (1-3) et forme les pâtons de pain avant de les enfourner (4-5); ils cuisent (6-8), puis sont tirés du four par des personnages indéterminés qui les disposent dans des paniers (9-10); des enfants se pressent sans doute pour les acheter (11). Voici le texte dans l'édition de Giovanni Salanitro ${ }^{12}$ :
Ipse manu patiens immensa uolumina uersat 1
adtollitque globos. Sonuerunt omnia plausu.
Tum Cererem corruptam undis emittit ab alto.
Septem ingens gyros, septena uolumina traxit,
lubrica conuoluens et torrida semper ab igni. 5
At rubicunda Ceres oleo perfusa nitescit.
Scintillae absistunt, opere omnis semita feruet.
Feruet opus redoletque, uolat uapor ater ad auras.
Instant ardentes ueribusque trementia figunt,
conclamant rapiuntque focis onerantque canistris. 10
Vndique conueniunt pueri innuptaeque puellae.

8 Dans le détail, chaque étape n'apparaît pas de manière absolument nette, et il y a certains mots, et surtout, certaines formes et certaines étapes du processus, à propos desquelles on peut hésiter : uolumina au v. 1 implique-t-il le sens précis de "rouleaux » ou est-il plus général ${ }^{13}$ ? De même, au v. 4, gyros apporte-t-il la précision que ces painsci ont des formes de couronnes plutôt que de boules ${ }^{14}$ ? Le sens que certains mots devraient trouver dans le contexte est encore moins clair : c'est surtout le cas de semita au v. $7^{15}$, mais aussi de torrida par rapport à lubrica au v. $5^{16}$, d'opus dans ses deux emplois, au v. 7 et $8^{17}$. Dans quelle mesure peut-on atténuer la valeur dépréciative du participe corruptam au v. 3 , surtout - et c'est au fond valable pour chaque segment - si l'on a en tête le contexte d'emploi dans Virgile (Aen. $\left.1,177^{18}\right)$ ?

Compte tenu de ces difficultés et en laissant autant que possible de côté les 'originaux' virgiliens et leurs contextes, je propose la traduction suivante :

Lui-même, de la main, patiemment, fait rouler des volumes immenses, puis les soulève en boules. Tout retentit sous le claquement des mains. Alors il laisse retomber Cérès mêlée d'eau.

Imposant, il en a tiré sept ronds, sept volumes, enserrant ces formes ondoyantes que la chaleur du feu saisit sans cesse. Mais alors Cérès baignée d'huile prend un 
éclat rougeoyant. Des étincelles jaillissent, le plancher $<\mathrm{du}$ four $>$ est tout bouillant pendant le travail. Le produit même est en effervescence et sent bon; une vapeur épaisse s'envole dans les airs.

On s'empresse avec ardeur, on embroche les pains encore tremblants. À grands cris, on les arrache aux foyers, et on en charge les corbeilles. De toutes parts accourent de jeunes garçons et des jeunes filles non mariées.

La singularité du texte vient de l'accent mis d'abord sur le travail de la pâte et la cuisson ; l'agitation finale, à la fois des ouvriers et des jeunes clients (voire des pains eux-mêmes, avec trementia, v. 8 !) renforce l'effet « scène de la vie de quotidienne » en permettant au lecteur de se la représenter plus aisément, peut-être, que ce qui précède. Mais la singularité de ce texte ne doit pas faire penser qu'il est le seul poème latin à parler de la fabrication du pain ${ }^{19}$. Un précédent important du De Panificio est un poème de la tradition virgilienne, que l'on ne considère généralement pas comme étant de Virgile, et que l'auteur du centon lui-même ne considère très probablement pas comme tel : c'est le Moretum ${ }^{20}$.

\section{Le précédent du Moretum}

11 La première partie 'culinaire' de ce poème de l'Appendice virgilienne est consacrée à la panification. Pour faire face au «triste jeûne » qui menace sa journée (v. 4), en effet, Simylus, le paysan protagoniste du poème, commence par préparer et cuire le pain auquel le moretum qui donne son nom à la pièce servira d'accompagnement. Mais le poète prend son sujet plus en amont que l'auteur du De Panificio: ici, la section prédominante est celle qui est consacrée à la mouture du grain (v. 16-43; les v. 31-38 sont cependant centrés sur Scybale, "gardienne»-custos, v. 31 - africaine, à qui Simylus demande de préparer le feu de cuisson et d'y mettre en attendant de l'eau à tiédir). Après avoir pris la quantité nécessaire de blé, disposé sa lampe près de la meule, nettoyé celle-ci, le paysan entreprend une tâche rendue plus rude par le fait qu'il l'accomplit entièrement à la main. De fait, les v. 24-29 sont le lieu d'une focalisation sur les mains du personnage :

Aduocat inde operi, partitus utrinque :

laeua ministerio, dextra est intenta labori. 25

Haec rotat adsiduum gyris et concitat orbem.

Tunsa Ceres silicum rapido decurrit ab ictu,

interdum fessae succedit laeua sorori

alternatque uices ${ }^{21}$.

Cette insistance se retrouve en partie dans la section ultérieure, quand Simylus passe la farine obtenue au crible et pétrit la pâte (manu, v. 39, v. 45, palmis, v. 47). Certains termes employés de façon récurrente peuvent faire le lien avec le De Panificio : il s'agit en particulier d'opus, vu ci-dessus au v. 24 dans le sens de "tâche ", et qui se retrouve ensuite au v. 39 pour désigner la meule (opus uersatile), et au v. 48 à propos de la pâte, "domptée », que le paysan amincit alors et étend sous la forme d'un rond ${ }^{22}$. Les emplois métonymiques de Cérès pour le pain peuvent être pris dans le même sens (d'autant que les deux emplois du centon, où Ceres est caractérisée par un participe ou un adjectif, peuvent correspondre aux deux premiers emplois - sur trois en tout - dans le Moretum $^{23}$ ) ; et il y a, enfin, unda au pluriel, employé pour l'eau qui, mêlée à la farine, va en faire de la pâte (Moretum, v. $44^{24}$, De Panificio, v. 3).

Leur sujet mis à part, les points de contact entre les deux textes, en somme, ne s'imposent pas avec l'évidence d'une allusion, à part peut-être au v. 3 du centon, dont le 
début et la fin semblent faire écho au v. 27 du poème de l'Appendix: Tunc Cererem corruptam undis demittit $\boldsymbol{a} \boldsymbol{b}$ al to / Tunsa Ceres silicum rapido decurrit $\boldsymbol{a} \boldsymbol{b}$ ictu. Or, en admettant la possibilité que l'auteur De Panificio connaisse le Moretum et même y fasse une référence consciente, et en considérant que, dans le Moretum, c'est l'action antérieure au travail de la pâte et à la cuisson du pain qui est le plus longuement détaillée, j'avancerai l'hypothèse que le début du centon, tel que nous l'avons (Ipse manu patiens immensa uolumina uersat), est recevable en tant que tel, avec un ipse qui peut s'entendre comme un ipse de succession - celle du boulanger du centon par rapport à Simylus faisant son pain dans le poème de l'Appendix ${ }^{25}$-, et que manu prend tout son sens du fait que, dans le Moretum, Simylus est amené à tout faire à la main : pétrir, mais aussi, et avant tout, moudre le grain. Le potentiel d'incipit que le premier vers du centon me semble ainsi posséder ${ }^{26}$ peut trouver un renfort supplémentaire dans le fait que les mots qui le composent se prêtent assez aisément à une lecture de type métapoétique qui tend à une affirmation de l'auteur ${ }^{27}:$ « Lui à son tour (sc. l'auteur du centon), patiemment, de sa main, roule et déroule les immenses rouleaux (contenant l'œuvre de Virgile) $»^{28}$. Un centoniste est, certes, fondamentalement, un homme de mémoire ${ }^{29}$ (qui, quand il crée, ne passe pas son temps à compulser son poète), mais il a vraisemblablement un jour lu et relu tout Virgile avant de commencer à en mémoriser un très grand nombre de vers ${ }^{30}$. Du reste, dans le Moretum aussi, les vers sur le pétrissage de la pâte se prêtent à une lecture en un sens poétique, surtout leuat opus au début du v. $48:$ il s'agit bien, dans ce poème, de présenter une forme "allégée » du maius opus épique (cf. en particulier le v. 59, quand Simylus passe à la préparation du moretum: Ergo aliam molitur opem sibi prouidus heros $\left.{ }^{31}\right)$, ou plutôt, de traiter un sujet quotidien et humble sur un rythme et en des mots souvent nobles ${ }^{32}$. C'est précisément ce que fait - Ipse manu patiens - le centoniste du De panificio, et de manière plus radicale encore, puisque ce rythme et ces mots nobles sont ceux de Virgile même. Mais ne s'agit-il que des mots?

\section{Du pain dans l'Énéide}

Dans le Codex Salmasianus un autre poème " culinaire » fait une belle place, sinon à la panification, du moins au pain, dont est fait un bref éloge : c'est le Iudicium coci et Pistoris iudice Vulcano (Le procès entre un cuisinier et un boulanger-pâtissier jugé par Vulcain) d'un certain Vespa, un vrai texte parodique, généralement daté du $4{ }^{\text {ème }}$ siècle, qui prend pour cible les controverses ${ }^{33}$. Un boulanger et un cuisinier s'opposent dans une joute oratoire où chacun défend les mérites et l'utilité de son travail. Le pâtissier parle le premier: après une invocation à Saturne, il loue le pain en tant que nourriture universelle et fondamentale : " n'est-il pas vrai que tout le monde a besoin de pain, que personne ne refuse? Car sans lui quelle sorte de repas les hommes pourraient-ils servir?» (v. 24-25) Suit une énumération d'exemples voués à en illustrer les vertus et le prestige. De façon remarquable, le dernier d'entre eux implique l'Énéide (v. 26-28) :

Qui uires tribuit, qui primum poscitur, hic est,

quem serit agricola, quem maximus educat aether.

Hunc pater Aeneas Troianis uexit ab oris ${ }^{34}$.

La substitution du panis aux Pénates est savoureuse ${ }^{35}$. Elle peut reposer sur la proximité entre les Penates et le penus, en tant que " garde-manger ", lieu à l'écart dans la maison, où sont stockées les provisions ${ }^{36}$. En outre, la référence faite par Vespa peut viser un épisode précis de l'Énéide: pour J.-Fr. Lespect ${ }^{37}$, il s'agit sans aucun doute des mensae 
auxquelles les Harpyes donnent un air si terrifiant au chant 3 de l'épopée de Virgile (v. 257), et dont on découvre au chant 7 que ce sont 'seulement' des mensae paniciae, des 'tables' en pain sacrées sur lesquelles les anciens Romains faisaient leurs libations aux dieux - nous apprend Servius à propos du v. 736 du chant 1 de l'Énéide ${ }^{38}$. Dans le passage fameux du chant 7 , certes en se conformant à la volonté de Jupiter ${ }^{39}$, les Troyens se servent surtout de galettes d'épeautre comme supports pour leur nourriture champêtre ${ }^{40}$, presque sauvage (des fruits, pomis agrestibus, v. 111) ${ }^{41}$ : ces galettes sont désignées de différentes manières, parmi lesquelles Ceres (exiguam in Cererem, v. 713), mais aussi adorea liba (v. 709), où liba fait entendre qu'il s'agit plus de gâteaux (sacrés et sucrés) que de galettes semblables à du pain ${ }^{42}$.

De fait, il me semble que l'auteur du Iudicium Coci et Pistoris pouvait avoir en tête un autre passage de l'Énéide où il s'agit, à proprement parler, de pain, et même, où est décrit le début d'un processus de panification : les v. 174-179, et plus particulièrement, les v. 177-179 du chant $1^{43}$.

Ac primum silici scintillam excudit Achates succepitque ignem foliis atque arida circum nutrimenta dedit rapuitque in fomite flammam.

Tum Cererem corruptam undis Cerealiaque arma expediunt fessi rerum, frugesque receptas

et torrere parant flammis et frangere saxo ${ }^{44}$.

D'après le commentaire servien ${ }^{45}$, le passage fut discuté par les anciens au moins à deux titres :

18 1. En ce qui concerne la panification même, certains lecteurs anciens devaient considérer le v. 179 comme fautif, parce qu'à leurs yeux, il présentait les opérations dans le désordre (la cuisson avant la mouture des grains, en somme). Une solution s'imposait, lire dans ce vers un hysteron-proteron, ce que refuse catégoriquement Servius ${ }^{46}$, qui avance plusieurs arguments: dans le contexte (où il a été dit que les grains ont été mouillés dans la tempête, Cererem corruptam undis, v. 177), torrere avant frangere fait sens, parce qu'il signifie précisément «faire sécher » (et non faire cuire); même en dehors de ce contexte, par exemple dans le passage des Géorgiques où l'on retrouve les deux verbes dans cet ordre (G. 1, 267 : nunc torrete igni fruges, nunc frangite saxo), la succession de ces deux actions est encore valide, d'ailleurs elle l'est encore du temps du commentateur (de son propre aveu ${ }^{47}$ ); du temps des ancêtres, ajoute ce dernier, il n'y avait pas de meules, et l'on faisait ainsi griller les grains avant de les piler dans un mortier (les meuniers, voire les boulangers, pistores, avaient d'abord été des " pileurs", pinsores) ${ }^{48}$.

19 2. L'inclusion d'une action ordinaire dans le carmen grande de Virgile pose un problème de convenance. Servius l'évoque d'abord à propos des mots choisis par le poète. Une expression comme Cerealiaque arma (Aen. 1,177$)$ montre, selon le commentaire, combien Virgile se garde d'entrer dans le détail de façon trop concrète, et veille au contraire à ennoblir par le langage d'humbles réalités. Il s'agit ensuite des actions en rapport avec la dignité des personnages, celle du héros en premier lieu : Énée reste à l'écart de cette première phase de préparation du repas que les Troyens las et probablement affamés vont pouvoir prendre ensuite (Aen. 1, 210-215). Lui va pourvoir à la seconde phase en fournissant la viande pour ce repas, après la chasse aux cerfs qu'il mène lorsqu'il s'éloigne de ses compagnons pour scruter la mer (Aen. 1, 180-193). Servius relève ainsi (à propos du v. 180, où le récit se détache des Troyens pour se concentrer sur Énée) le soin que Virgile met partout à ne pas faire participer les 
personnes de valeur aux tâches ordinaires, pour ne pas dire aux basses corvées ${ }^{49}$. Or ce passage n'est pas le seul où l'intrusion de l'humile dans le grande et surtout la façon dont Virgile assume et 'gère' cette intrusion sont discutées à propos d'un repas ${ }^{50}$. De fait, le repas des Troyens sur le rivage carthaginois n'est pas le seul repas de l'Énéide.

\section{Manger dans l'Énéide (aperçu)}

20 L'alimentation et la cuisine forment un sujet qui été relativement peu traité pour l' Énéide, et même plus généralement, pour l'ensemble de l'œuvre de Virgile. Cela tient sans doute, en partie, à des raisons que j'avançais en commençant : un sujet si bas (qui se trouve de fait illustré de façon bien plus fréquente dans des œuvres soi-disant mineures ou liées à la réalité quotidienne ${ }^{51}$ ) ne semble guère compatible avec une œuvre si grande. Pourtant, on sait que Virgile n'a pas reculé devant les humilia, en particulier dans ses deux premières œuvres où il revendique de donner l'illustration qu'ils méritent aux objets 'ténus' qui peuplent ces poèmes; et dans l'Énéide même, l'humilité - de certains personnages et de leur manière d'être, notamment - est parfois ce qui assure à la vraie grandeur de naître. Naturellement, il y a des degrés dans l' humile ou le uile, et il y a des limites que Virgile n'a manifestement pas voulu dépasser : si Bucoliques et Géorgiques ${ }^{52}$ peuvent chacune fournir une sorte de 'recette' (un moretum dans Buc. 2, 10-11, du vin cuit en G. 1, 295-6 $6^{53}$ ), ce n'est tout de même pas le cas de l' Énéide ${ }^{54}$; là, quand Virgile se risque à reprendre à Homère une de ces 'vignettes' domestiques qui, dans l'Iliade surtout, charment tant, c'est en se gardant d'en conserver les éléments les plus 'crus' : un exemple est la graisse remplacée par de l'eau, dans la comparaison du chant 7 à propos de Turnus (Aen. 7, 462-466), rendu (encore plus) bouillant par Allecto ${ }^{55}$. Cela étant, la thématique alimentaire est bien présente dans l' Énéide ${ }^{56}$, et il est d'ailleurs possible de l'aborder à plusieurs niveaux.

Dans son ouvrage De la table des dieux à la table des hommes. La symbolique de l'alimentation dans l'Antiquité romaine ${ }^{57}$, Inês de Ornellas e Castro a eu le grand mérite de consacrer une partie importante à l'Énéide, en posant fondamentalement la question de savoir comment Virgile était parvenu à intégrer dans son maius opus ce thème «bas » de la nourriture et de la cuisine. Sa réponse principale est convaincante, même si, à vrai dire, peu surprenante (parce qu'elle me semble ancrée dans le genre épique, dans la tradition de l'épopée homérique où cette 'règle' vaut déjà $\left.{ }^{58}\right):$ les passages où Virgile aborde le plus directement le sujet alimentaire, à savoir les cinq repas qu'il met en scène dans le poème (à Carthage, sur le rivage et auprès de Didon; en Sicile; lors de l'arrivée en Italie ; à Pallantée $)^{59}$, sont presque tous ${ }^{60}$ soumis à la logique de la « cuisine du sacrifice "; dans leur organisation et leur composition, ils remplissent les conditions du 'bon' repas, civilisé et pieux, qui contribue à rapprocher les hommes des dieux. Dans ce cadre-là, le sacrifice importe plus que la cuisine. Et lorsque l'on mange dans l'Énéide (lorsqu'il est dit que les Troyens mangent dans l'Énéide), c'est essentiellement pour supprimer la faim ${ }^{61}$.

D'ailleurs, j'ajouterai que la faim est un thème 'montant' dans le poème : c'est même un topos naissant, à en juger par sa récurrence dans les épopées ultérieures ${ }^{62}$. La prophétie menaçante de Celaeno au chant 3 (v. 247-257) se vérifie quasiment à la lettre ; pour la guerre qu'ils provoquent - ici (aux Strophades) et là (dans le Latium), bon gré mal gré (dans les deux cas) - les Troyens 'méritent' la faim. De fait, un choix remarquable de Virgile consiste à avoir exclu le traitement direct de la thématique alimentaire des 
chants guerriers de son épopée, de sa partie proprement iliadique : il n'y a plus de repas dans les chants 9 à 12 de l'Énéide ${ }^{63}$. Mais il y a des images saisissantes, de combats qui deviennent sanglants comme de la viande crue (deiecta crudescit pugna Camilla, Aen. 11, 83364), d'arme qui goûte le corps de celui qui qu'elle frappe ([lancea] summum degustat uolnere corpus, Aen. 12,376 ${ }^{65}$ ), et aussi d'un héros qui se remplit de la vue du baudrier de son jeune ami défunt jusqu'à ce que la colère s'empare de lui de manière irrépressible (Aen.12,945-6: oculis postquam saeui monimenta doloris / exuuiasque hausit ${ }^{66}$ ), tandis qu'une déesse jusqu'alors insatiable (voir en particulier Aen. 5, 608: [Saturnia Iuno] multa mouens necdum antiquum saturata dolorem ${ }^{67}$, et 781 : Iunonis grauis ira neque exsaturabile pectus ${ }^{68}$ ) est présentée comme pouvant être elle-même 'mangée' par son dolor (ne te tantus edit tacitam dolor ${ }^{69}$, Aen. 12, 801).

En somme, l'exploration des traitements virgiliens du thème de l'alimentation peut et doit sans doute être prolongée. Ici, il va s'agir de voir ce que l'auteur du De panificio a fait de cette 'réalité' virgilienne.

\section{Le centon et son projet : une parodie de Virgile?}

Dans la lecture que je vais donner du centon, je n'entends pas nier qu'il a parfois pour effet (et peut-être, pour visée) de faire rire ou au moins sourire. Je ne veux pas non plus nier que le fait d'appliquer tel segment de vers de l'épopée virgilienne qui représente celle-ci dans ce qu'elle a de plus noble (un épisode de combat, par exemple) à la fabrication de pains constitue un abaissement qui tend à l'héroï-comique ${ }^{70}$. Mon intention est avant tout de souligner que le centoniste a une bonne, voire une très bonne, connaissance de l'épopée et de l'art de Virgile, et que c'est ce qui lui a permis d'y prélever des extraits qui s'accordent avec son poème au sujet humble. De fait, la plupart des passages qu'il cite ne semblent pas choisis au hasard, ou seulement au gré des associations d'images ou de sons qui jouent un rôle si important dans ce genre de créations $^{71}$. Certes, la présence de scintillae absistunt, au début du v. 7 , est peut-être surtout due à un enchaînement phonique qui s'est produit dans l'esprit - et la mémoire - du centoniste, par rapport au segment précédent, dans la deuxième partie du v. 6, qui s'achevait sur nitescit. De même, lubrica conuoluens, souvenir-éclair de Pyrrhus comparé à un serpent au chant 2 de l'Énéide (Aen. 2, 471-475), doit peut-être de se trouver au début du v. 5 au fait que l'auteur avait puisé, pour le vers précédent, - septem ingens gyros, septena uolumina traxit - dans un locus de sa mémoire peuplé par les serpents de Virgile $^{72}$.

Sans doute n'est-ce pas pour une raison absolument différente que la "Cérès rougeoyante " (At rubicunda Ceres) de G.1,297, figure, en début du v. 6, après une citation du même poème (et torrida semper ab igni, G. 1, 234) : rougeoiement et chaleur torride lient en effet les deux extraits (on trouve rubens - semper sole rubens - dans la première partie du v. 234, et le v. 298 contient une forme de torrere : et medio tostas aestu terit area fruges); mais on peut imaginer en outre que le centoniste savait très bien ce qu'il y avait avant le At du v. 297 : une évocation de l'hiver et de ses travaux, avec un tableau domestique - un regard jeté sur la vie privée des humbles - assez rare chez Virgile et d'autant plus mémorable (G. 1, 293-296) ${ }^{73}$.

interea longum cantu solata laborem

arguto coniunx percurrit pectine telas, 
aut dulcis musti Volcano decoquit umorem

et foliis undam trepidi despumat aeni ${ }^{74}$.

Des deux activités de l'épouse qui sont présentées dans ces vers, la cuisson du moût est, du reste, celle qui contribue le plus à la « rareté » de la scène. Pour celle-ci, enfin, R.F. Thomas n'exclut pas la possibilité d'une inspiration callimachéenne, et plus précisément, de l'Hécalé ${ }^{75}$. Or il me semble que la remémoration de lieux semblables à ce passage des Géorgiques a pu servir au centoniste de principe de composition, ou tout du moins, de fil directeur dans l'invention de son poème.

\section{L'enseignement du premier vers}

Cette hypothèse se vérifie dès le début du De Panificio, avec le premier segment cité : la reprise d'un passage épique lui-même écrit sur un mode mineur - qui soulève de nouveau la question de l'humile dans le graue -, avec une discrète attention portée au thème alimentaire. Ipse manu patiens est une citation modifiée (ipse remplace ille, manu remplace manum ${ }^{76}$ ) d'Aen. 7, 490, un vers qui appartient à l'ekphrasis du cerf de Silvia, avec ille qui se rapporte au cerf. On sait à quel point cette invention de Virgile fit réagir et même déplut dans l'Antiquité: dans l'épisode de Cyparissis, Ovide exacerbe le raffinement des soins donnés au cerf et souligne le caractère disproportionné de la douleur que sa mort suscite ${ }^{77}$; dans les Saturnales, Macrobe regrette que Virgile n'ait pas su inventer un début plus noble pour la guerre dans le Latium, autrement dit, qu'il n'ait pas su trouver un modèle homérique auquel se conformer ${ }^{78}$.

Mais le caractère si particulier, 'anormal' du passage n'est sans doute pas la seule raison pour laquelle le centoniste l'a choisi ${ }^{79}$ : rappeler le souvenir de ce cerf pouvait être un moyen de faire allusion aux sept autres, i.e. aux cerfs dont la viande forme une part importante du premier repas de l'Énéide. En outre, cette allusion n'est pas absolument nécessaire pour le lecteur qui sait se souvenir de la suite du v. 490 du chant 7 de l'Énéide : le mot qui suit patiens, en effet, n'y est autre que mensa (ille manum patiens mensaeque adsuetus erili ${ }^{80}$ ). Immensa, au début du segment repris ensuite, s'est peut-être imposé pour l'écho qu'il produit avec ce 'mot-clef' absent - mensa - auquel il assure malgré tout une vraie présence. Alors que la première partie du vers ne semble pas vraiment porter atteinte à la gravité de Virgile ou à la dignité épique (pas plus que Virgile lui-même n'a paru l'avoir fait dans l'épisode du cerf), la deuxième partie de ce v. 1 peut illustrer l'effet de parodie que produit de temps à autre, et / ou sur certains lecteurs, ce texte ${ }^{81}$. Et cet effet est sans doute prolongé au début du v. 2.

Dans les deux cas, en effet, le souvenir de créatures énormes, gigantesques - Éryx, d'un côté $^{82}$, l'Etna de l'autre ${ }^{83}-$, plane potentiellement sur la figure du boulanger, qui en ressort grandi à l'extrême. Mais dans un tel cas, comme à l'échelle du centon en son entier, est-ce que ce qui doit primer, est la dégradation que cela implique pour les vers de Virgile, ou est-ce plutôt l'élévation (par la langue de Virgile) de la matière (la boulange) que le poète a choisi d'aborder? Comme le souligne Scott McGill, chaque lecteur peut réagir de manière différente et interpréter de manière différente un centon et ce centon en particulier ${ }^{84}$. De mon point de vue, c'est la deuxième configuration qui prime, et au-delà du choix de passages fait par le centoniste, je serais tentée d'en trouver un indice supplémentaire dans les mots mêmes de la première partie du v. 2, adtollitque globos, «il soulève les boules <de pâte> » : adtollo, en effet, peut et pourrait y signifier « élever » dans un sens figuré (i.e. grandir, glorifier) et constituer 
ainsi un commentaire sur l'opération entreprise par l'auteur du De Panificio. C'est une forme d'élévation superlative, excessive, et l'on peut se rappeler que, aux yeux de certains lecteurs antiques, le passage de Virgile impliqué par cette citation péchait précisément par un tel défaut stylistique ${ }^{85}$.

Mais je ne pense pas que ce soit le motif qui ait poussé le centoniste vers la description de l'Etna : celle-ci contient en fait plusieurs mots évocateurs de l'acte de manger (voire de trop manger), comme lambit, à la fin du v. 574, eructans au v. 576 ${ }^{86}$; le nom globus luimême désigne une «boule » dont la nature est spécifiée par le génitif qui le complète (flammarum dans Virgile, farinae sous-entendu dans le centon), mais c'est aussi, au pluriel, le nom d'un mets romain simple et fréquent ${ }^{87}$. Il est alors possible que le centoniste ait exacerbé l'intérêt de Virgile pour la chose alimentaire et / ou gastronomique, en relevant des passages qui n'en parlent pas directement, mais qui comportent des termes susceptibles de l'évoquer ${ }^{88}$. Voyons maintenant à quel point, plus simplement (de façon moins partiale ${ }^{89}$ ), il a rendu présente la 'cuisine' de Virgile dans son texte.

\section{Le De Panificio et la 'cuisine' de Virgile}

31 L'auteur a en fait soigneusement évité de faire défiler les passages de l'Énéide faisant le plus directement intervenir la nourriture, à savoir les repas dont il a été question plus haut. Toutefois, de façon très significative, il a retenu quelques segments de vers représentant le premier et le dernier de ces repas, avec, aux deux extrémités, une évocation plus ou moins implicite du sujet du centon, à savoir le pain même: le (premier) repas en Libye se trouve rappelé deux fois, lors de sa préparation, avec Aen. 1, 177, Cererem corruptam undis, au v. 3, puis lors de son déroulement, avec Aen. 1, 212, ueribusque trementia figunt, au v. 9 ; le repas chez Évandre est ensuite rendu présent au v. 10 avec Aen. 8,180, onerantque canistris - le complément d'objet du verbe onerare dans Virgile n'étant autre que dona laboratae Cereris (Aen. 8, 181). Certain autre repas semble figurer dans le centon de manière discrète, mais non moins efficace: l'épisode fameux des tables peut être d'autant plus contenu dans im-mensa au v. 1 que le début du vers fait peut-être lui-même allusion à la conclusion dudit épisode au chant $7^{90}$.

Deux repas ou séquences de repas ont donc visiblement été laissés de côté par l'auteur : ce sont le banquet en Libye (où le luxe fait oublier ce que l'on mange ${ }^{91}$ ) et les repas en Sicile, lors des cérémonies en l'honneur d'Anchise. Toutefois, dans ce second cas, le centoniste semble avoir opté pour une solution tout à fait intéressante : délaissant les festins des hommes, il a vraisemblablement préféré penser à un 'goûter' animal. Le v. 4 du centon cite en effet le début de la scène de l'apparition du serpent, lorsqu'Énée a commencé le rituel, fait les libations, adressé une prière à son père mort et à ses Mânes ; et ce rituel n'est mené à bien que lorsque le serpent a achevé son tour, du fond du tombeau d'Anchise aux autels dressés jusqu'au tombeau de nouveau: les sacrifices et le banquet qui s'ensuit ne sont accomplis qu'une fois que le serpent s'est nourri, en goûtant lui-même aux offrandes (Aen. 5, 90-94) ${ }^{92}$.

Obstipuit uisu Aeneas. Ille agmine longo

tandem inter pateras et leuia pocula serpens

libauitque dapes rursusque innoxius imo

successit tumulo et depasta altaria liquit.

Hoc magis inceptos genitori instaurat honores ${ }^{93}$ 

sensations produites par la cuisson des pains -, le centoniste fait intervenir des passages pertinents de Virgile. Là où il aurait pu, pour faire franchement rire, filer la métaphore du fournil assimilé à un volcan (cf. adtollitque globos au v. 2) et identifier ses acteurs aux Cyclopes forgerons, il s'en est bien gardé95. Mais il a donné juste ce qu'il faut pour rendre néanmoins l'image présente à l'esprit du lecteur: feruit opus redolentque au début du v. 8 rappelle en effet la description de l'effervescence dans la ruche en G. 4, 158 sqq., où le labeur si intense des abeilles leur vaut d'être effectivement comparées aux Cyclopes (G. 4,170 sqq.). Cette comparaison, qui accroît encore la distance entre la petitesse du sujet du chant et la grandeur des moyens mis en œuvre pour le traiter, peut relever de la dimension héroï-comique que l'on a parfois reconnue à cette quatrième Géorgique ${ }^{96}$ : dès la première partie de celle-ci, et de façon plus nette qu'auparavant, Virgile traite régulièrement son sujet comme si c'était un sujet d'épopée (martiale : on peut de fait penser en particulier à un passage comme le combat des rois aux v. 67 sqq.).

L'auteur du De Panificio connaît sans doute cette particularité du texte de Virgile, en vertu de laquelle son propre texte est bien moins éloigné et 'en-dessous' de celui-ci qu'il ne pourrait sembler ${ }^{97}$. Et l'auteur doit savoir en outre que feruit opus redolentque et ce passage sur l'intense labor des abeilles $(G .4,162-169)$ ont été repris par Virgile dans l' Énéide (Aen. 1, 431-436) à propos des Carthaginois tout occupés à l'édification de leur 
ville : un autre extrait du même passage du chant 1 de l'épopée se trouve au v. 9 (instant ardentes, Aen. 1, 423, avant la comparaison avec les abeilles et la reprise des vers de la quatrième Géorgique).

En outre, le passage du chant 4 qui comporte une comparaison similaire - celle des Troyens à des fourmis travailleuses - est aussi représenté dans le centon, par opere omnis semita feruet au v. 7 ; or la façon dont ce passage (Aen. 4, 402-407) est écrit (et dont les insectes sont représentés) rappelle d'assez près - par certains termes, mais surtout par le ton, par l'esprit - la description des abeilles dans la Géorgique 4.
Ac uelut ingentem formicae farris aceruom
cum populant hiemis memores tectoque reponunt,
it nigrum campis agmen praedamque per herbas
conuectant calle angusto ; pars grandia trudunt
obnixae frumenta umeris, pars agmina cogunt
castigantque moras, opere omnis semita feruet ${ }^{98}$.

Faute de contextes, il reste difficile d'avoir un avis définitif sur la reprise par Virgile, au v. 404, d'un it nigrum campis agmen dont Servius (ad loc.) nous apprend qu'Ennius l'avait employé à propos des éléphants et Accius des Indiens : mais le «sourire secret » que R.G. Austin prête à Virgile en considérant cette description des fourmis me parait, en tout cas, très plausible : « grandia, trudunt, obnixae, umeris all show Vergil with a secret smile, as he smiles at his bees in Georgics $4^{99}$ ". L'auteur du De Panificio me semble partager ce sourire avec son modèle : son texte reste fondé sur un écart - Virgile n'a nulle part traité aussi directement d'un sujet aussi domestique, aussi quotidien, aussi concret, que la fabrication du pain -; mais cet écart est visiblement réduit de différentes manières ou pour différentes raisons : parce que le centoniste manifeste sa conscience que la poésie de Virgile n'est cependant pas fermée aux thèmes de l'alimentation et de la cuisine ; parce qu'il connaît l'intérêt de Virgile pour les lieux et les sujets humbles; mais donc aussi parce qu'il s'appuie sur des passages où Virgile luimême a travaillé sur des écarts, en particulier entre un contenu et un style, tendant parfois à l'héroï-comique.

\section{Le projet du De Panificio}

40 Un ultime constat, fort intrigant, reste à présenter. Il semble s'agir également d'un élément à part entière du programme ou du projet du De Panificio; pour ce qui l'a motivé, d'autres explications pourront être données au-delà des propositions que je vais faire. Si l'on s'intéresse aux parties précises de la poésie de Virgile (aux œuvres et aux chants au sein de celles-ci) que le centoniste a le plus sollicitées, on trouve que le chant 5 de l'Énéide fournit le plus grand nombre de citations. Mais ce n'est pas tout : ces citations proviennent de chacun des différents épisodes qui composent le chant 5 :

\begin{tabular}{|l|l|}
\hline Vers du De Panificio et vers de Virgile concerné & Episode correspondant du chant $\mathbf{5}$ \\
\hline v. 4, Aen. 5, $85:$ septem ingens gyros, septena uolumina traxit & Rites en l'honneur d'Anchise \\
\hline \hline v. 6, Aen. 5, 135: oleo perfusa nitescit & Régate \\
\hline v. 11, Aen. 5, 293 : undique conueniunt & Course à pied \\
\hline
\end{tabular}




\begin{tabular}{|l|l|}
\hline v. 1, Aen. $5,408:$ immensa uolumina uersat & Combat de ceste \\
\hline v. 2, Aen. 5, $506:$ sonuerunt omnia plausu & Tir à l'arc \\
\hline v. 10, Aen. 5, $660:$ conclamant rapiuntque focis & Incendie des vaisseaux \\
\hline
\end{tabular}

41 Il manque seulement un rappel du ludus Troianus. Cette absence ne remet pas en cause le caractère systématique de ces citations prises dans un même chant, et de manière suffisamment organisée pour distinguer les parties qui le composent. À considérer ces citations, on pourrait voir le centon comme une sorte de version miniature de l'Énéide 5. Qu'est-ce qui a pu pousser le centoniste à cela? En allant de la moins à la plus satisfaisante, $\mathrm{j}$ 'avancerais prudemment les hypothèses suivantes: le chant 5 est spécialement mis en avant en tant que chant de la Sicile, terre particulièrement riche en grains, matière première du pain ; le chant 5 est le chant de l'Énéide que le centoniste connaissait ou appréciait le mieux; le chant 5 est le plus festif de l'épopée virgilienne, qui associe les banquets aux Jeux. Ceux-ci en font le chant «ludique » de l'Énéide, ce qui le rend le plus proche du ludus que constitue fondamentalement la composition d'un centon ${ }^{100}$ et du ludus dont relève, tout au plus, à mon sens, ce centon.

Le De Panificio devait-il faire rire pour être un centon réussi ? Ou serait-ce un centon raté par la proximité que, selon ma lecture, il instaure ou rétablit entre son texte et celui de Virgile ${ }^{101}$ ? Une différence de fond reste, et elle est incontestable: Virgile n'a jamais illustré, dans les vers nobles qui sont, en général, les siens, une telle scène de la vie quotidienne ; il n'a jamais décrit aussi longuement une opération domestique aussi concrète que la fabrication du pain ${ }^{102}$. À ce titre, de l'œuvre de Virgile - et surtout de l' Énéide, le poème le plus cité - au poème De Panificio, un détournement et une forme d'abaissement sont bien en jeu, en principe. En pratique, par les segments de Virgile qu'il a adoptés, l'auteur de ce centon nous suggère que Virgile n'était pas loin d'écrire un texte comme le sien, que son œuvre, même l'Énéide, contenait une telle potentialité. Il ne lui a d'abord pas échappé que l'Énéide contenait une scène de panification, et il connaissait les autres passages de l'épopée illustrant la thématique alimentaire. Le recours qu'il y a fait pourrait, certes, être vu comme le fruit d'une technique mémorielle (relativement) basique, consistant à s'appuyer sur des ressemblances de situation $^{103}$. Mais l'auteur ne s'est pas contenté de cela, il a cité de tels passages avec jugement ${ }^{104}$ et discrétion, et il leur a associé d'autres passages susceptibles de renforcer sa démarche : d'une part, des loci virgiliens où il n'est pas question de nourriture ou de cuisine, mais où des mots peuvent les évoquer ${ }^{105}$; d'autre part, des loci où Virgile avait exploré les rapports et la distance entre les styles, parfois les genres, notamment en introduisant des figures de l'humilité dans son maius opus (le cerf de Silvia), ou en s'appliquant à grandir des humilia (les abeilles). Dans cette mesure, la valeur de succession que j'ai prêtée au ipse liminaire - s'il devait être le vrai seuil du centon devrait passer, non plus entre l'auteur du Moretum et le centoniste, mais bel et bien entre Virgile et ce « successeur » qui, à son tour, lit et relit d'immenses volumes, et qui a à cœur d'honorer, dans ses vers, les modestes sujets. 


\section{Annexe : Le centon 'décomposé'}

\section{Le centon et ses 'intertextes' virgiliens}

Ipse manu patiens (Aen. 7, 490 [ille manum]) immensa uolumina uersat (Aen. 5, 408)

Adtollitque globos.(Aen. 3, 574) Sonuerunt omnia plausu. (Aen. 5, 506)

Tunc Cererem corruptam undis (Aen. 1, 177 [tum]) emittit ab alto. (Aen. 1, 297 [demittit])

Septem ingens gyros, septena uolumina traxit (Aen. 5,85$)$

lubrica conuoluens (Aen. 2, 474 [conuoluit]) et torrida semper ab igni. (G. 1, 234)

At rubicunda Ceres $(G .1,297)$ oleo perfusa nitescit (Aen. 5, 135)

Scintillae absistunt $($ Aen. 12, 102) opere omnis semita feruet $($ Aen. 4,407$)$

Feruet opus redoletque (G. 4, 169 [redolentque]) uolat uapor ater ad auras (Aen. 7, 466)

Instant ardentes (Aen. 1, 423) ueribusque trementia figunt (Aen. 1, 212)

Conclamant rapiuntque focis $($ Aen. 5,660$)$ onerantque canistris $($ Aen. 8,180$)$

Vndique conueniunt (Aen. 5, 293 [ou 9,720]) pueri innuptaeque puellae ( G. 4,476 [ou Aen. 6, 307])

\section{Les fragments utilisés et leur contexte d'origine}

1. Aen. 7, 490 (cerf de Silvia), contaminé avec Aen. 7, 143 (ipse manu quatiens, à propos de Jupiter, lors du prodige des tables) : Ille manum patiens mensaeque adsuetus erili / errabat siluis...

Aen. 5, 407-408 (Jeux, ceste): magnanimusque Anchisiades et pondus et ipsa / huc illuc uinclorum immensa uolumina uersat.

2. Aen. 3, 574-576 (Etna) : attollitque globos flammarum et sidera lambit / interdum scopulos auolsaque uiscera montis / erigit eructans...

Aen. 5, 506 (Jeux, tir à l'arc): Intremuit malus timuitque exterrita pennis / ales, et ingenti sonuerunt omnia plausu.

3. Aen. 1, 177-179 (Libye, fabrication du pain): Tum Cererem corruptam undis Cerealiaque arma / expediunt fessi rerum, frugesque receptas / et torrere parant flammis et frangere saxo.

Aen. 1, 297 sqq. (Libye, Jupiter envoie Mercure sur terre): Haec ait et Maia genitum demittit ab alto / ut terrae utque nouae pateant Karthaginos arces / hospitio Teucris, ne fati nescia Dido / finibus arceret.

4. Aen. 5, 84-6 (cérémonie religieuse à la mémoire d'Anchise, le serpent) : Dixerat haec, adytis cum lubricus anguis ab imis / Septem ingens gyros, septena uolumina traxit / Amplexus placide tumulum lapsusque per aras

5. Aen. 2, 474-5 (Pyrrhus comparé à un serpent) : lubrica conuoluit sublato pectore terga / arduos ad solem, et linguis micat ore trisulcis.

G. 1, 234 (les parties du monde): Quinque tenent caelum zonae : quarum una corusco / semper sole rubens et torrida semper ab igni.

6. G. 1, 297 (de l'hiver à l'été, les travaux) : At rubicunda Ceres medio succiditur aestu / et medio tostas aestu terit area fruges.

Aen. 5, 134-5 (Jeux, préparatifs pour la régate) : Cetera populea uelatur fronde iuuentus / nudatosque umeros oleo perfusa nitescit. 
7. Aen. 12,102 (Turnus): His agitur furiis tototque ardentis ab ore / scintillae absistunt, oculis micat acribus ignis, / mugitus ueluti cum prima in proelia taurus / terrificos ciet...

Aen. 4, 402-407 (comparaison pour les Troyens se préparant à quitter Carthage) : ac uelut ingentem formicae farris aceruum / cum populant hiemis memores tectoque reponunt, / it nigrum campis agmen praedamque per herbas / conuectant calle angusto; pars grandia trudunt / obnixae frumenta umeris, pars agmina cogunt / castigantque moras, opere omnis semita feruet.

51 8. G. 4,169 sqq. $(4,169$, intense activité des abeilles = Aen. 1,436 , intense activité des Carthaginois) : Feruit opus,redolentque thymo fragrantia mella. / Ac ueluti lentis Cyclopes fulmina massis / Cum properant, alii taurinis follibus auras / Accipiunt redduntque, alii stridentia tingunt / Aera lacu...

Aen. 7, 460-6 (Turnus comparé à un chaudron d'eau bouillante) : arma amens fremit, arma toro tectisque requirit; / saeuit amor ferri et scelerata insania belli, / ira super: magno ueluti cum flamma sonore / uirgea suggeritur costis undantis aeni / exsultantque aestu latices, furit intus aquai / fumidus atque alte spumis exuberat amnis, / nec iam se capit unda, uolat uapor ater ad auras.

9. Aen. 1, 423-4 (intense activité des Carthaginois quand Énée les aperçoit): instant ardentes Tyrii: pars ducere muros / molirique arcem et manibus subuoluere saxa, / pars optare locum tecto et concludere sulco;

Aen. 1, 212 (Libye, premier repas, la viande) : Pars in frusta secant ueribusque trementia figunt

53 10. Aen. 5, 660 (les Troyennes sur le point d'incendier les vaisseaux): Tum uero attonitae monstris actaeque furore / conclamant rapiuntque focis penetralibus ignem.

Aen. 8, 179-181 (Pallantée, banquet): Tum lecti iuuenes certatim araeque sacerdos / uiscera tosta ferunt taurorum onerantque canistris / dona laboratae Cereris Bacchumque ministrant.

11. Aen. 5, 293 (Jeux, rassemblement pour la course à pied) $=9,720-1$ (les Latins 'inspirés' par Mars, épisode de Pandarus) : Vndique conueniunt Teucri mixtique Sicani Vndique conueniunt, quoniam data copia pugnae / bellatorque animo deus incidit.

G. 4, 475-7 (foule des morts aux enfers) = Aen. 6, 306-8 (foule des morts aux enfers): matres atque uiri defunctaque corpora uita / magnanimum heroum, pueri innuptaeque puellae / Impositique rogis iuuenes ante ora parentum

\section{BIBLIOGRAPHIE}

R.G. Austin, P. Vergili Maronis Aeneidos liber quartus, edited with a commentary, Oxford, 1982

M. Bažil. Centones Christiani : Métamorphoses d'une forme intertextuelle dans la poésie latine chrétienne de l'Antiquité tardive, Paris, Collection des Études Augustiniennes : Série Moyen Âge et Temps Modernes 47, 2009

A. Blossier-Jacquemot, « Le Cento Nuptialis d'Ausone ou le mariage de Virgile », Mosaïque 3 (revuemosaique.net), mars 2010, p. 109-142 
I. de Ornellas e Castro, De la table des dieux à la table des hommes. La symbolique de l'alimentation dans l'Antiquité romaine, Paris, L'Harmattan, 2011

F. Ermini, Il centone di Proba e la poesia centonaria latina, Roma, 1909

S. Galhac, « Boire et manger dans l'Iliade et l'Odyssée », dans Actes des Journées d'études Boire et manger, 4-6 avril 2011, Université de Savoie, à paraître

E. Gowers, The loaded table. Representations of Food in Roman Literature, Oxford, 1993

P. Hardie, Rumour and Renown. Representations of 'Fama' in Western Literature, Cambridge University Press, 2012

S. Hinds, Allusion and Intertext, Cambridge, 1998

S. Hinds, «The self-conscious cento », dans M. Formisano and T. Fuhrer eds., Décadence: 'Decline and Fall' or 'Other Antiquity', Heidelberg, Winter, à paraître.

N. Horsfall, Virgil, Aeneid 7. A commentary, Leiden, Brill, 2000

N. Horsfall, « The Moretum Decomposed », C\&M 52, 2001, p. 308-318

N. Horsfall, Virgil, Aeneid 11. A commentary, Leiden, Brill, 2003

E.J. Kenney, Moretum: a poem ascribed to Virgil, Bristol, Classical Press, 1984

J.-F. Lespect, « Le Iudicium Coci et Pistoris iudice Vulcano (Anthologie Latine 199), introduction, traduction et notes », Folia Classica Electronica (Louvain-la-Neuve), numéro 9, janvier-juin 2005

R.O.A.M. Lyne, Words and the Poet. Characteristic Techniques of Style in Vergil's Aeneid, 1998

S. McGill, Virgil recomposed.The Mythological and Secular Centos in Antiquity, Oxford University Press, 2005

N. Monteix, "L'art du pain dans l'Antiquité romaine ", dans Le blé, l'autre or des Romains, Exposition présentée au musée archéologique départemental à Bavay, du 17 mars au 31 août 2010, Bavay, Conseil général du Nord, 2010, p. 42-48.

N. Monteix, Les lieux de métier : boutiques et ateliers d'Herculanum, Rome, EFR, 2010

R.A.B. Mynors, Virgil. Georgics, Oxford, 1990

D.O. Ross, «The Culex and Moretum as Post-Augustan Literary Parodies », HSCPh 79, 1975,

p. $235-263$

G. Salanitro, «Osidio Geta a la poesia centonaria », ANRW 2.34.3, Berlin, 1997, p. 2314-p. 2360

G. Salanitro, Silloge dei Vergiliocentones minori. Introduzione, testo critico, traduzione e note, Roma, Bonanno editore, 2009.

R.F. Thomas, Virgil, Georgics, volume 1, books I-II, volume 2, books III-IV, Cambridge University Press, 1988

G. Tronchet, «Corolles pour Narcisse. Une lecture ovidienne au cœur d'un centon virgilien », Dictynna [en ligne] 7, 2010

\section{NOTES}

1. Josse Bade (Iodocus Badius Ascensius), Opera Vergiliana docte et familiariter exposita..., Paris, Jean Petit, 1512. Une version plus développée de l'anecdote se trouve déjà dans la Vita Donati aucti, 8-18 (p. 80-84 Vitae Vergilianae antiquae Brugnoli-Stok). 
2. Le centon nous est parvenu sans titre, De panificio lui a été donné comme titre, exempli gratia, par les éditeurs. F. Ermini, Il centone di Proba e la poesia centonaria latina, Roma, 1909, en tient compte (p. 42). Voir G. Salanitro, "Osidio Geta a la poesia centonaria », ANRW 2.34.3, Berlin, 1997, p. 2314-p. 2360 (p. 2336).

3. L'ouvrage de Scott McGill, Virgil recomposed.The Mythological and Secular Centos in Antiquity, Oxford University Press, 2005, est d'une lecture très stimulante et a certainement joué un rôle moteur dans la reprise des études sur le centon. Parmi les études récentes centrées sur un centon, je citerai celles d'Anne Blossier-Jacquemot, «Le Cento Nuptialis d'Ausone ou le mariage de Virgile », Mosaïque 3 (revuemosaique.net), mars 2010, p. 109-142, et de Gilles Tronchet, « Corolles pour Narcisse. Une lecture ovidienne au cœur d'un centon virgilien », Dictynna [en ligne] 7, 2010. Je me permettrai de renvoyer à ces différents travaux pour une ample bibliographie.

4. C'est l'édition de R.D. Shackleton Bailey, Anthologia Latina, I, 1, Stuttgart, Teubner, 1982. (Les premiers mots de la Préface sont consacrés à ce rejet des centons.) L'édition usuelle (pour tous les centons "mythologiques» sauf celui d'Ausone) reste celle d'A. Riese (Anthologia Latina, I, 1, Leipzig, Teubner, 1869), où le De Panificio se trouve p. 23. Dans l'édition qu'il prépare pour la CUF, Daniel Vallat intègre bien ces centons.

5. Les trois études citées (n. 2) décrivent les règles de composition du centon.

6. Sur ce centon et sur ce point, voir en particulier A. Blossier-Jacquemot, «Le Cento Nuptialis d'Ausone... ", p. 120-122.

7. Les deux poèmes sont humbles en général ou par principe, mais comme les commentateurs anciens le soulignaient déjà, les Bucoliques n'ignorent pas l'élévation; et ennoblir les humilia est un défi clairement exprimé dans les Géorgiques.

8. Cf. Sc. McGill, Virgil recomposed..., p. 55.

9. Sc. McGill, Virgil recomposed..., p. 54, donne comme trait définitoire de la parodie le fait qu'elle «dégrade le matériau de départ pour produire un effet comique." De son point de vue, les centons De panificio et De alea (ainsi que les sections conclusives de l'Epithalamum Fridi et du Cento Nuptialis d'Ausone) se distinguent des autres parce qu'ils ne se contentent pas de modifier le matériau virgilien, mais qu'ils le rabaissent, étant donné leur sujet (le pain, le jeu de dés, le rapport sexuel). Sc. Mc Gill intègre ensuite (p. 57 sqq.) à son analyse du De Panificio une discussion de sa nature " parodique », et il précise pour finir (p. 63-64) que, d'après lui, cette parodie n'a pas de visée critique au sens où il ne s'agit ni d'attaquer Virgile ni de pointer (pour le railler) un aspect particulier de sa poésie; en se donnant pour but de 'dégonfler' (angl. « deflate ») Virgile en employant ses vers pour parler de la fabrication du pain, le centoniste viserait la poésie de Virgile même, en tant que poésie « canonique » (op. cit., p. 60).

10. Les centons chrétiens constituent l'autre tradition, très importante, de centons virgiliens : à leur propos, voir notamment l'étude récente de M. Bažil. Centones Christiani: Métamorphoses d'une forme intertextuelle dans la poésie latine chrétienne de l'Antiquité tardive, Paris, Collection des Études Augustiniennes: Série Moyen Âge et Temps Modernes 47, 2009. L'auteur aborde utilement, pour commencer, la question théorique des rapports entre les centons et l'intertextualité.

11. Ces dates extrêmes sont les dates de composition supposées de la Médée d'Hosidius Geta et de l'Epithalamium Fridi de Luxorius.

12. Silloge dei Vergiliocentones minori. Introduzione, testo critico, traduzione e note, Roma, Bonanno editore, 2009. Je redonne en annexe le texte du centon assorti des références aux vers cités de Virgile.

13. Des scènes antiques de pétrissage présentent des rouleaux de pâte (voir par exemple la fig. 5695, reproduction d'un groupe de pétrisseuses en terre cuite provenant de Thèbes, p. 496 du Dictionnaire des Antiquités grecques et romaines de Daremberg et Saglio, t. IV, vol. 1, art. pistor, M. Besnier). Il me semble que les mouvements dépeints au début du centon, surtout aux v. 2-3, évoquent davantage le geste du pizzaiolo pétrissant sa pâte, laquelle forme dès lors plus un «volume », une «masse » qu'un « rouleau » : l'artisan l'élève au-dessus de son plan de travail, 
donne des coups fermes de ses mains, la laisse retomber, et ce, à plusieurs reprises. Sur d'autres représentations, la pâte façonnée a une forme plus ronde qu'allongée : voir par exemple le relief de boulangerie du Museo Civico archeologico de Bologne présenté par Nicolas Monteix, «L'art du pain dans l'Antiquité romaine ", dans Le blé, l'autre or des Romains, Bavay, 2010, p. 43 et 44. Cet article fournit une synthèse précise sur la panification dans l'Antiquité ; une étude plus longue et détaillée se trouve dans N. Monteix, Les lieux de métier : boutiques et ateliers d'Herculanum, Rome, EFR, 2010, chapitre III, « Boulangeries et confection du pain.»

14. Nicolas Monteix, que je remercie pour l'attention qu'il a prêtée à mon travail et à mes questions, confirme que la variété des formes de pains dans l'antiquité romaine permet une telle hésitation, que cette forme de couronnes est en outre possiblement attestée à Herculanum même.

15. Je comprends qu'il s'agit ici de la sole, c'est-à-dire du " plancher » du four. Nicolas Monteix (per litteras) me suggère plutôt de lire semita comme le « sillage » des braises que l'on sort du four avant d'y placer les pains. Ainsi, la cuisson à proprement parler ne serait mentionnée qu'au v. 8, tandis qu'au v. 6 serait évoquée une première coloration des miches à l'ouverture du four.

16. J'ai opté pour une opposition entre la mobilité (le caractère glissant) et le raidissement (en l'occurrence, sous l'effet du feu).

17. Je suppose que de l'un à l'autre il y a un léger glissement de sens, du processus au produit (dans le deuxième cas, surtout parce qu'opus est aussi sujet de redolet).

18. Il s'agit de l'après-tempête, nous y viendrons plus loin dans le détail. Corruptam peut renvoyer à la transformation en jeu dans le passage de l'état de blé/farine à celui de pâte : c'est ce qui est décrit aux v. 44-45 du Moretum dont il sera également question plus bas.

19. On suppose l'existence d'une littérature sur pain et gâteaux à l'époque alexandrine. Le pain et d'autres produits céréaliers étaient abordés au début de l'Hedypatheia, le poème gastronomique d'Archestratos de Gela (fr. 5-6 Olson-Sens). Dans la discussion sur le sujet aux chapitres 109A-113C du livre 3 des Deipnosophistes, Athénée mentionne plusieurs auteurs de traités sur le pain et la fabrication du pain, dont Chrysippus de Tyane.

20. Sc. Mc Gill, Virgil recomposed..., p. 58, mentionne le Moretum pour souligner l'extrême brièveté du centon en comparaison de ce texte où la description des actes, la présentation du cadre, la caractérisation des personnages, sont bien plus fournies. En renvoyant aux travaux de E.J. Kenney (Moretum : a poem ascribed to Virgil, Bristol, Classical Press, 1984) et de D.O. Ross ("The Culex and Moretum as Post-Augustan Literary Parodies ", HSCPh 79, 1975, p. 235-263) il exclut (n. 26 p. 190) la possibilité d'une relation directe entre le De Panificio et le Moretum, parce que, même si les dates le permettent (pour le Moretum, il reprend l'hypothèse de D.O. Ross, qui en fait un poème de l'époque de Tibère : voir aussi E.J. Kenney, p. xxii-xxvii, et N. Horsfall, « The Moretum Decomposed », C\&M 52, 2001, p. 308-318, p. 305), il n'y a pas, dans l'Antiquité, de mention de ce poème, qui n'apparaît dans le corpus virgilien qu'au $9^{\text {ème }}$ siècle (Kenney, Ibid., p. xxi).

21. "Alors il fait appel à ses deux mains, partageant le travail de part et d'autre. La gauche est vouée au service, la droite au gros du travail. Elle tourne en cercles incessants et emporte le cylindre. Cérès broyée tombe en courant sous le choc rapide des pierres. De temps en temps la main gauche remplace sa sœur fatiguée et alterne avec elle. »

22. De fait, "pâte" (ou même "pain») pourrait être la traduction commune des deux occurrences d'opus dans le centon.

23. Tunsa Ceres au v. 27, emundata Ceres au v. 43. La troisième occurrence se trouve aux v. 54-55, quand Simylus entreprend de cuisinier le fromage avec les herbes, « de peur que Cérès seule ne fasse pas l'agrément de son palais. "

24. Voir E.J. Kenney ad loc., p. 27, qui présente cet emploi comme ovidien plus que virgilien.

25. Cela ne réglerait sans doute pas tout le problème posé par ce ipse initial : mais on sait que les poètes latins jouent parfois de ce pronom fortement «identitaire ", en l'employant par exemple dans des contextes où l'identité du ou des personnages en cause n'est pas claire : je pense au 
«epic ipse» repéré par Ph. Hardie, Rumour and Renown. Representations of 'Fama' in Western Literature, Cambridge University Press, 2012, p. 200 sqq. D’ailleurs, dans le vers concerné de Virgile (Aen. 7, 490), on ne lit pas ipse mais ille; et le sujet de uersat en Aen. 5, 408 (la source pour la deuxième moitié du v. 1 du centon) n'est autre que le héros «lui-même ", Énée (désigné par magnanimusque Anchisiades au v. 407, vers conclu par le mot ipsa se rapportant aux uolumina).

26. Je remercie vivement Daniel Vallat d'avoir bien voulu me donner per litteras les précisions suivantes: le De Panificio occupe le haut de la première page du Codex Salmasianus, sans indication de lacune, mais aussi sans titre. Or on sait qu'il manque des quaternions au début du manuscrit. Pour que le texte commence vraiment comme in medias res (et sans plus de précision relativement à ipse) sur le v. 1 tel que nous l'avons, il faut imaginer le titre occupant la toute dernière ligne de la page précédente. Ce cas de figure se rencontre à plusieurs reprises dans le manuscrit (Sacrilegus capite puniatur, A.L. 21 Riese, début du titre en 47r, texte en $47 \mathrm{v}$, Verba amatoris, ibid. 23, titre en $58 \mathrm{v}$, texte en $59 \mathrm{r}$, de incesto partu, ibid 70 , titre en $64 \mathrm{v}$, texte en $65 \mathrm{r}$, de capone, ibid. 132, titre en $86 \mathrm{v}$, texte en $87 \mathrm{r}$, de balneis, ibid. 175 , titre en $94 \mathrm{v}$, texte en $95 \mathrm{r}$ ). Si l'hypothèse d'une lacune liminaire devait être maintenue, le nombre de vers moyen des centons courts fait penser qu'elle n'est pas très longue.

27. La propension des centons à la réflexivité a été montrée et amplement illustrée par Stephen Hinds, à partir des centons de Proba et d'Ausone, dans une communication ("The self-conscious cento ») présentée lors du colloque Entre imitatio et intertextualité. Citation et allusion dans la poésie latine, organisé par V. Berlincourt, L. Galli Milic et D. Nelis à la Fondation Hardt, Vandoeuvres, Genève, les 31 octobre et $1^{\mathrm{er}}$ novembre 2011. Ainsi que le souligne S. Hinds, dans la façon que les centonistes ont de commenter leur démarche, leur méthode de composition et ses fruits, ou encore dans la sphragis qu'ils insèrent çà et là dans leurs créations, se manifeste une conscience d'auteur, ou tout du moins, une conscience poétique qui contribue à montrer que, pour eux, ce jeu littéraire n'était pas sans dignité ou ambition. Cette étude de $\mathrm{S}$. Hinds paraîtra bientôt, avec le même titre ("The self-conscious cento ») dans M. Formisano and T. Fuhrer, eds., Décadence: 'Decline and Fall' or 'Other Antiquity', Heidelberg, Winter.

28. Pour cette application du verbe uersare, «tourner, faire tourner », voir par exemple Horace, A.P., v. 268-269 : Vos exemplaria Graeca / nocturna uersate manu, uersate diurna (« Vous, feuilletez de jour, feuilletez de nuit les modèles grecs »). Volumen est notamment employé par Ovide à propos des Métamorphoses (Tr. 1, 1, 117 = 3, 14, $19 ; \operatorname{Tr} .1,7,35$ ).

29. Au début de sa Lettre-préface, Ausone définit le centon comme étant un travail de la seule mémoire (solae memoriae negotium, Cento Nuptialis, Praef. 3). Voir en outre Sc. McGill, Virgil recomposed..., p. 10-11.

30. L'auteur du centon se présenterait d'abord dans la posture d'un lecteur ; ce faisant, il peut aussi renvoyer une image de ses propres lecteurs, tels qu'il se les représente; mais la question de la façon dont les centons étaient reçus demeure presque entière. Et un centoniste attendait-il vraiment de son lecteur qu'il aille vérifier, au besoin, la provenance des segments de Virgile (en l'occurrence) qu'il avait mobilisés ? De fait, la lecture que j'ai pu faire du centon et dont je rendrai compte dans la deuxième partie de cette étude est une lecture 'patiente', attentive au détail des contextes virgiliens, dont je n'ai pas les moyens de dire si un lecteur ancien l'aurait pratiquée de la même manière. En ce qui concerne une éventuelle figuration de la réception du poème, je signalerai la proposition que m'ont faite des étudiants de master avec qui j'ai travaillé sur le centon : dans omnia sonuerunt plausu au v. 2 ils ont perçu une possible référence au public, (par avance) enthousiaste, du centoniste.

31. "Notre héros prévoyant se ménage donc un autre secours. "

32. E. Gowers, The loaded table. Representations of Food in Roman Literature, Oxford, 1993, p. 46-47, souligne le lien entre création culinaire et création poétique dans le poème; l'insistance sur la pauvreté, la petitesse et, par ailleurs, la variété, peut être lue comme l'affirmation d'une poétique. 
33. Sc. McGill, Virgil recomposed..., mentionne ce texte (p. 59) et écrit qu'il comporte une parodie de compétition de chant bucolique.

34. "Celui qui donne des forces, celui que l'on demande en premier, c'est lui, lui que le paysan sème, que l'éther très haut fait lever. C'est lui que le vénérable Énée a transporté des bords de Troie. »

35. Cf. notamment Aen. 1, 378-379: Sum pius Aeneas, raptos qui ex hoste Penates / classe ueho mecum... ( Je suis le pieux Énée, je transporte avec moi sur ma flotte les Pénates arrachés à l'ennemi...»).

36. Mais le pain ne fait pas partie des denrées non périssables qui forment le penus, par contraste avec le cellarium ou la cella, où sont entreposées les denrées à consommer sous peu de jours : voir la note dans le commentaire de Servius ad Aen. 1, 703. Aulu-Gelle 4,1 offre une discussion sur le sens exact de penus et dans la définition de Q. Scaevola rapportée par Favorinus au paragraphe 17 , on retrouve la distinction évoquée.

37. J.-Fr. Lespect, « Le Iudicium Coci et Pistoris iudice Vulcano (Anthologie Latine 199), introduction, traduction et notes », Folia Classica Electronica (Louvain-la-Neuve), numéro 9, janvier-juin 2005, « Introduction » : http://bcs.fltr.ucl.ac.be/fe/09/VespaIntro.html

38. Servius ad Aen. 1, 736 : LATICVM LIBAVIT HONOREM more sacrorum: et tangit ritum Romanorum, qui panicias sacratasque mensas habebant, in quas libabant, ut est 'heus etiam mensas consumimus, inquit Iulus'. (" selon la coutume des cérémonies : il évoque un rite des Romains, qui avaient des tables en pain consacrées sur lesquelles ils faisaient des libations, comme dans 'hé ! nous mangeons même nos tables', dit Iule »)

39. Sic Iuppiter ipse monebat (v. 110).

40. Servius ad Aen. 7, 111, explique l'emploi de solum (Cereale solum) dans ce contexte et dans ce même vers : cela peut se dire de tout ce qui soutient quelque chose; c'est pourquoi Virgile l'emploie ici à propos des «tables en pain, à savoir le support des mets » (sic nunc panicias mensas, id est epularum sustentaculum, solum uocauit).

41. Pour cette occurrence unique de pomum dans le poème et sur ce repas particulier, voir Inês de Ornellas e Castro, De la table des dieux à la table des hommes, Paris, 2011, p. 56, p. 90-91.

42. Servius ad Aen. 7, 109 : ADOREA LIBA ador proprie est genus farris, liba autem sunt placentae de farre, melle et oleo, sacris aptae («ador, 'l'épeautre', est à proprement parler une sorte de froment, tandis que liba, 'les gâteaux sacrés', sont des galettes à base de froment, de miel et d'huile, faites pour les cérémonies sacrées »).

43. Sc. McGill, Virgil recomposed..., p. 62, fait le même lien.

44. "Avant tout, Achate a fait jaillir l'étincelle d'un silex, il a recueilli le feu sur des feuilles, tout autour il a mis des aliments bien secs et il a aussitôt fait prendre feu aux copeaux. Alors, quoiqu'épuisés par les événements, ils préparent Cérès gâtée par l'eau, ainsi que les ustensiles de Cérès : ils s'apprêtent à griller sur les flammes et à broyer sous la pierre les grains qu'ils ont sauvés. "

45. Il s'agit de la longue note à propos d'Aen. 1, 179 .

46. Tiberius Claudius Donat (ad loc.) fait de même dans ses Interpretationes Vergilianae.

47. Servius ad Aen. 1, 179: Nam et hodie siccari ante fruges et sic frangi uidemus (« de fait, aujourd'hui même, nous voyons que les grains sont asséchés et ainsi, broyés »).

48. Servius auctus ajoute (entre autres choses) une sorte d'argument supérieur qui veut que la série des opérations mentionnées par Virgile soit conforme au droit pontifical. Les compagnons ont agi comme l'auraient fait des flamines, pour remédier à la «corruption » du blé par l'eau, mais surtout, pour veiller à ce que la préparation ne fermente pas : ils ne pouvaient entrer en contact avec une farine fermentée (cf. la Question romaine 109 de Plutarque).

49. AENEAS merita personarum uilibus officiis interesse non debent; quod bene seruat ubique Vergilius, ut hoc loco ( « les personnes de valeur ne doivent prendre part aux tâches vulgaires, ce que Virgile observe, à juste titre, partout, comme dans le présent passage »). 
50. Ainsi, dans la première partie du v. 701 (dant manibus famuli lymphas), lors du banquet de Didon, Servius relève la présence du style humble (humilis character), en accord avec ce qui est alors décrit : les actions des serviteurs donnant de l'eau... et du pain (Cereremque canistris).

51. C'est ce que souligne très justement E. Gowers (The loaded table..., p. 22), qui mentionne aussi les textes «mixtes", "pratiquant le mélange », et qui délimite ainsi le corpus sur lequel son étude est centrée.

52. Où l'humilitas peut être 'enfreinte' ou dépassée (qu'on pense par exemple aux Bucoliques 4-6), et où elle est aussi très contrôlée, pour ce qui est surtout de l'expression et du traitement dans le détail de certains sujets.

53. Voir, à propos de ce passage, les précisions apportées par R.A.B. Mynors (Virgil. Georgics, Oxford, 1990), ad loc., p. 68. L'inclusion de recettes dans un ouvrage sur l'agriculture est critiquée dans Varron, R.R. I, 2,28, avec le cas précis des recettes culinaires contenues dans le De agricultura de Caton.

54. À propos du mets préparé par Thestylis dans la Bucolique 2, N. Horsfall ("The Moretum decomposed », p. 306) fait cependant la remarque qu'il ne déroge pas à l'élégance de cette poésie bucolique, dès lors que le gros, la partie la plus consistante du repas de Simylus - le pain - est omis.

55. Cf. Il. 21, 361 sqq. et voir N. Horsfall, Virgil. Aeneid 7..., ad 462-6, p. 310 : il suppose que la graisse apparaissait comme quelque chose de trop bas selon le goût augustéen.

56. Elle l'est aussi dans les Bucoliques et les Géorgiques, sur lesquelles je ne m'attarderai pas, à ce titre, ici : c'est vers l'Énéide que les deux textes auparavant abordés (Moretum et Iudicium Coci et Pistoris) m'ont guidée, et l'Énéide est l'œuvre de Virgile de loin la plus utilisée par le centoniste du De Panificio (il n'y a pas de citation des Bucoliques, et seulement trois segments proviennent exclusivement des Géorgiques). Les centonistes privilégient en général l'Énéide.

57. Paris, L'Harmattan, 2011.

58. Sur la façon dont boire et manger sont traités dans les épopées homériques, voir l'étude toute récente de S. Galhac, "Boire et manger dans l'Iliade et l'Odyssée », à paraître dans les Actes des Journées d'études Boire et manger, 4-6 avril 2011, Université de Savoie. La dimension rituelle est également prédominante dans les épopées homériques. La pietas si importante dans l'Énéide peut, il est vrai, engendrer une différence de degré. Il est également vrai que Virgile parvient à innover au sein du cadre et du modèle fournis par Homère.

59. Il s'agit des repas auxquels les Troyens participent; je laisse de côté ici la diète barbare d'un Polyphème. Sur ce genre de repas (que l'auteur inscrit dans la catégorie de la « table de l'autre »), voir Inês de Ornellas e Castro, De la table des dieux..., p. 50 sqq.

60. Inês de Ornellas e Castro (De la table des dieux...) montre de façon convaincante que même quand ils ne le semblent pas (parce que les circonstances ne le permettent pas), les repas des Troyens suivent le schéma du repas religieusement ordonné: la démonstration est particulièrement intéressante dans le cas du premier repas du chant 1 que nous avons déjà rencontré. La chercheuse montre (p. 80-81) que, même s'il repose pour une large part sur la chasse, ce repas est rendu traditionnel et rituel parce que Virgile fait finalement figurer la triade sacrificielle (du pain, du vin et de la viande) et compense l'absence d'un sacrifice en bonne et due forme par des éléments comme la mention des aena au v. 213 (ces chaudrons peuvent suggérer la cuisson que les Troyens vont bel et bien veiller à opérer sur les parties réservées aux dieux - les exta - tandis qu'ils font rôtir pour eux les uiscera). La table qui échappe le plus à ce juste modèle est au fond la table de Didon (op. cit., p. 82 sqq.), où les apparences (le décor, le personnel, la vaisselle...) prennent en définitive trop de place. Mais cela ne la rend certes pas indigne de l'épopée.

61. Cf. I. de Ornellas e Castro, De la table des dieux..., p. 73.

62. Voir notamment Ovide, Met. 8,777-842, Lucain, B.C. 4, 94 sqq. (Ilerda, épisode qui fait aussi intervenir la soif, v. 292 sqq.), Silius, Punica, 2, 463 sqq. 
63. Les 'déplacements' virgiliens sont ici remarquables : chez Homère, les repas ne manquent pas dans l'Iliade et c'est même dans l'Iliade que l'on trouve une scène de 'cuisine' tout à fait étonnante, où la préparation du mets (de la viande) semble l'emporter sur la dimension rituelle : en Il. 9 , 206-221, où l'on apprend comment la viande est découpée, mise en broche, cuite et salée en cours de cuisson. Par ailleurs, comme le montre Sylvie Galhac dans l'étude citée («Boire et manger dans l'Iliade et l'Odyssée ", cf. supra, n. 58) la faim apparaît plutôt comme un thème odysséen.

64. "Sur Camille tombée le combat devient plus sanglant». N. Horsfall (Virgil, Aeneid 11. A commentary, Leiden-Boston, 2003, p. 440) fait le lien non seulement avec Aen. 7, 788 où crudesco se trouve également (effuso crudescunt sanguine pugnae), mais aussi avec l'emploi d'acerbus à propos de la blessure ("âpre ») de Camille en Aen.11, 823. Ce dernier emploi illustre la façon dont les «adjectifs qui habituellement désignent les saveurs du palais» constituent plutôt « l'assaisonnement de la narration », comme le dit Inês de Ornellas e Castro (De la table des dieux..., p. 73) : ils sont séparés des moments de prise de nourriture et réservés pour un registre figuré.

65. «[La lance] effleure [litt. déguste] le corps d'une blessure. »

66. «Après qu'il eut absorbé du regard ces ornements, témoins d'une douleur cruelle.»

67. «[la Saturnienne Junon] qui remue maintes idées et n'a pas encore rassasié son antique ressentiment. »

68. « la lourde colère de Junon et son cœur insatiable. » Sur le jeu de mots étymologique dans ces emplois - Saturnia mis en rapport avec des adjectifs de la famille de satur - et sur exsaturabile en particulier, voir R.O.A.M. Lyne, Words and the Poet. Characteristic Techniques of Style in Vergil's Aeneid, 1998, p. 174 sq. L'auteur traite aussi (Ibid., p. 56 sqq.) l'emploi de degusto en Aen. 12, 376.

69. "de peur qu'une si grande rancœur te ronge en silence."

70. L'emprunt le plus efficace de ce point de vue est, selon moi, celui d'emittit (demittit chez Virgile) $a b$ alto en Aen. 1, 297 : le contexte virgilien est celui du premier envoi de Mercure par Jupiter à Carthage. Le segment de vers virgilien est alors véritablement détourné, et ce détournement est renforcé par le fait que, malgré le changement radical de contexte, le complément d'objet d'emittit au v. 3 du centon porte encore un nom de divinité : celui de Cérès (Cererem corruptam undis) ; le geste du boulanger tient alors d'une parodie de scène (d'envoi) de messager épique.

71. Sc. McGill, Virgil recomposed..., p. 12 sqq. décrit précisément les moyens mnémotechniques usuellement mis en œuvre par les auteurs de centons dans leur travail de composition.

72. D'ailleurs, il y a une probable contamination avec G.3,426 où on lit squamea conuoluens sublato pectore terga (à propos du serpent de Calabre), tandis que le vers de l'Énéide présente la forme conjuguée conuoluit, au lieu de conuoluens (lubrica conuoluit sublato pectore terga, Aen. 2, 474, «il déroule son corps glissant, dresse sa poitrine »). Il n'est pas rare qu'un centoniste combine deux débuts de vers virgiliens pour le fragment qu'il retient: un exemple se trouve au v. 6 du centon Narcisse ; voir G. Tronchet, « Corolles pour Narcisse » [supra n. 2], p. 56. G. Salanitro, Silloge dei Vergiliocentones..., p. 59, n. 5, précise cependant que la variante conuoluens est donnée dans le manuscrit Veronensis XL pour le vers de l'Énéide.

73. Toute cette séquence des Géorgiques apparaît comme un lieu virgilien très 'fréquenté' : le tableau que l'on vient d'évoquer contient le détail du chant par lequel l'épouse du paysan adoucit son travail (G. 1, 293), un motif traditionnel que l'on retrouve par exemple dans le Moretum, v. 30); G. 1, 299 est également fameux pour la variante parodique qu'un détracteur ancien de Virgile en a proposée (Nudus ara, sere nudus, habebis frigore febrem - au lieu de hiems ignaua colono : voir Vita Donatiana, $\S 43,1.7-8$, p. 39 Brugnoli-Stok). Par ailleurs, la possibilité que l'auteur du centon travaille sur les contextes élargis des segments qu'il mobilise me semble renforcée dans le cas présent par le fait que, dans les vers qui suivent le vers mobilisé, G. 1, 197, vient une nouvelle évocation de la saison hivernale, avec d'abord les moments festifs qu'elle contient : ceux-ci sont illustrés par une comparaison avec la navigation, le retour des navires au port. Or le segment que 
le centoniste a ensuite mobilisé au v. 6, oleo perfusa nitescit provient du chant 5 de l'Énéide, et précisément, de l'épisode de la régate.

74. «Pendant ce temps, apaisant de son chant un labeur qui se prolonge, son épouse fait courir sur la toile un peigne fin, ou bien, sur le feu de Vulcain, elle fait réduire la douce liqueur du moût et écume avec des feuilles l'onde du chaudron qui bout ».

75. R.F. Thomas, Virgil, Georgics, volume 1, books I-II, Cambridge University Press, 1988, ad G. 1, 295-296, p. 118-119. R.F. formule cette hypothèse d'un souvenir de l'Hécalé en considérant les similitudes entre la 'vignette' de Virgile et l'épisode de Philémon et Baucis dans les Métamorphoses (8, 641-650 en particulier).

76. Certains manuscrits virgiliens portent néanmoins, à cet endroit, la leçon manu. Ipse manu se rencontre six fois dans l'œuvre de Virgile, dont quatre fois en tête de vers (il existe néanmoins des variantes pour $G .3,395$ et Aen. 2,645 ) : assez près dans le chant 7 , se trouve ainsi ipse manu quatiens ostendit $a b$ aethere nubem, v. 143, où ipse désigne Jupiter («l'agitant lui-même de sa main, il fait voir du haut de l'éther une nuée » : il s'agit du présage favorable qui couronne l'épisode de la manducation des tables... et transforme celle-ci en un joyeux festin (Aen. 7, 146-147). La 'correction' et le choix effectués par le centoniste pour ce début de v. 1, censé reprendre le v. 490 du chant 7 de l'Énéide, pourraient alors favoriser une allusion à l'épisode de l'œuvre que l'on associe le plus spontanément à l'alimentation, l'épisode des « tables».

77. Ovide, Met. 10, 106-142, avec en particulier le v. 133 : ut leuiter pro materiaque doleret / admonuit [Phoebus](« Phébus l'engagea à verser des pleurs légers, proportionnés à leur objet »).

78. Macrobe, Sat., 5, 17, 1-4.

79. L'ekphrasis du cerf de Silvia a aussi été évaluée en termes de genre, et on y a détecté des échos de l'élégie, et aussi de la bucolique. D'une manière générale, le récit de l'éclatement de la guerre au chant 7 de l'Énéide propose un (re-)parcours de l'univers pastoral à l'univers épique. Plus spécifiquement, l'ekphrasis du cerf accueille un ensemble d'allusions aux Bucoliques, la troisième et la dixième en particulier (cf. e.g. Buc. 3, 94-97 et Aen. 7, 489, 494-495, Buc. 3, 93 et Aen. 7, 505, via G 3, 419, Buc. 10, 59-60 et Aen. 7, 497) ; ces allusions rappellent que le monde bucolique n'était pas à l'abri de tout danger, qu'au contraire, il comportait des menaces. En tout cas, mobiliser ce passage du chant 7 peut être ainsi un moyen indirect, pour le centoniste, de convoquer aussi le premier opus virgilien.

80. «Lui, qui se laissait caresser, habitué à la table de ses maîtres "

81. Par " parodie »,j'entends toujours, suivant le développement de Sc. McGill une réécriture qui abaisse le matériau-source ou le texte-modèle dans une visée comique : cf. supra n. 9.

82. Immensa uolumina uersat se trouve au v. 408 du chant 5 de l'Énéide: c'est Énée qui soupèse alors les cestes gigantesques qu'Entelle, acceptant le combat, vient de jeter à terre. Entelle est supposé avoir été l'élève d'Éryx, demi-frère d'Énée, de qui ces cestes proviennent.

83. Adtollitque globos ouvre le v. 574 d'Énéide 3, au début de la description de l'Etna, dont Virgile, on le sait, fait la prison d'Encélade (Aen. 3, 578 sqq.).

84. Sc. McGill, Virgil recomposed..., p. 63.

85. Voir Aulu-Gelle, 17, 10, 10 sqq.

86. L'Etna « vomit » les entrailles de la montagne (uiscera montis, Aen. 3, 575).

87. Voir Varron, L.L. 5, 107, Caton, Agr. 79, 1. Les globi sont aussi un des plats romains les plus aisés à préparer aujourd'hui et connaissent un certain succès auprès des collégiens latinistes qui ont la chance de pratiquer le latin en cuisine (et le latin tout court)!

88. Le début du v. 10 (conclamant rapiuntque focis) peut être aussi concerné par cette hypothèse : les femmes troyennes enlevant aux foyers le feu pour en incendier les navires (Aen. 5, 660) peuvent être vues comme pervertissant un geste domestique et pacifique consistant à retirer des aliments du feu qui les a cuits.

89. La possibilité que le centoniste s'approprie des passages de Virgile en leur donnant une 'saveur' gastronomique - un intérêt pour la nourriture - qu'ils n'avaient pas à l'origine, rappelle 
les appropriations et autres lectures tendancieuses analysées par S. Hinds dans Allusion and Intertext (Cambridge, 1998).

90. Voir la note 76 supra.

91. Voir I. de Ornellas e Castro, De la table des dieux..., p. 82-85 et surtout p. 84. Seuls le pain et le vin sont mentionnés, respectivement au v. 701 et au v. 724 du chant 1 . Le centoniste aurait pu recourir au v. 701 (pour la mention Cereremque canistris), mais Aen. 8, 180 (onerantque canistris) lui permettait d'être plus allusif, d'atteindre le dernier repas de l'Énéide, et d'éviter, finalement, un banquet trop pompeux pour son texte.

92. Dans cette séquence, I. de Ornellas e Castro, De la table des dieux..., p. 87, précise que les sacrifices accomplis par Énée et le banquet qui suit (Aen. 5, 100-103) doivent être distingués parce que les premiers, les suouetaurilia d'animaux noirs, constituent un holocauste aux dieux Mânes (toutes les pièces de bétail étaient donc consumées par le feu). Le récit fait néanmoins s'enchaîner les 'repas'.

93. «En le voyant, Énée fut frappé de stupeur. Lui, en une lente progression, rampa pour finir au milieu des patères et des coupes polies, le serpent ; il goûta aux plats sacrés puis il s'en retourna au fond du tombeau, abandonnant les autels où il s'était nourri. Énée n'en reprend que mieux les cérémonies entreprises en l'honneur de son père. »

94. Cf. N. Horsfall, Virgil, Aeneid 7..., p. 310, ad Aen. 7, 462-6: «V, we shall see, is at great pains to exalt his scene from the hearth in the highest language $»$.

95. Cf. Sc. McGill, Virgil recomposed..., p. 59, où l'auteur précise que la parodie offerte, selon lui, par le De Panificio, ne vise pas une réalité virgilienne particulière, ou une scène spécifique, ou un livre, ou un poème... Il donne les Cyclopes en exemple: "Thus the breadmakers are not comic doubles of the Cyclopes toiling in the smithy in A. 8.423-453, for example ».

96. La notion d'héroï-comique («mock-heroic ») est explicitement repoussée par R.F. Thomas parce qu'elle implique un humour qui, de son point de vue, est un effet que ce poème en particulier risque constamment, mais surtout si on l'envisage comme un tout, évite (je paraphrase ce qu'il écrit à la fin de sa note ad G. 4,3, Virgil, Georgics, volume 2, Books III-IV, Cambridge University Press, 1988, p. 148). «Comique » est peut-être, en effet, gênant, dans ce terme composé qui, dans sa définition usuelle, décrit pourtant très bien le dessein de Virgile dans la première moitié de cette quatrième Géorgique : chanter en un style élevé (à la manière épique) un sujet qui se voit caractérisé tour à tour par les adjectifs leuis, tenuis, angustus. Mais si Virgile n'a pas écrit cette Géorgique pour faire rire, cela n'interdit pas de l'imaginer souriant devant sa création et les disproportions qu'elle comporte.

97. Par « en-dessous ", je pense à l'explication de Sc. McGill selon laquelle, à la différence des autres centons, dans le De Panificio et le De alea, les changements apportés aux vers de Virgile ne se feraient pas sur un plan horizontal, mais plutôt sur un plan vertical : voir Virgil recomposed..., p. 54 et 55 .

98. «Ainsi, lorsque des fourmis, à la pensée de l'hiver, pillent un énorme tas de blé et le mettent à l'abri sous leur toit, elles vont, en colonne noire, à travers les plaines et sur un sentier étroit, elles transportent leur butin parmi les herbes. Les unes mettent toutes leurs forces pour pousser de leurs épaules des grains immenses, d'autres ferment la marche et blâment les retardataires, leur travail met tout le chemin en effervescence. »

99. R.G. Austin, P. Vergili Maronis Aeneidos liber quartus, edited with a commentary, Oxford, 1982, p. 125, adAen. 4, 404 sqq.

100. Voir Sc. McGill, Virgil recomposed..., p. 5 sqq. (et p. 54 en relation avec le De Panificio vu comme une parodie là où les autres centons relèvent seulement du ludus/lusus). La notion de jeu (ludus, ludere) apparaît à trois reprises dans la présentation qu'Ausone fait du centon dans sa lettre-préface à Paulus. 
101. Je prends l'occasion de ces questions - qu'ils ont soulevées - pour remercier les participants au séminaire de l'Institut d'études anciennes de l'Université Laval, dans le cadre duquel j'ai eu la chance de pouvoir présenter une première version de ce travail.

102. Les vers du chant 1 de l'Énéide qui décrivent un début de panification et dont Cererem corruptam undis,au v. 3 du centon, est extrait, sont cependant très remarquables pour leur précision relative : I. de Ornellas e Castro (De la table des dieux..., p. 58) insiste sur cette précision, « unique dans le poème ». Du reste, la précision n'est sans doute pas la principale qualité du De Panificio: je renvoie aux alternatives mentionnées plus haut, en rapport avec la traduction proposée ; certains gestes ou événements continuent malgré tout d'intriguer : emittit ab alto, opere omnis semita feruet... Par cet aspect, même si ce n'est pas le premier texte visé par l'auteur, le centon se rapprocherait plutôt des Géorgiques, où bien des opérations concrètes sont décrites, mais d'une manière qui fait dire à Servius, au début de son commentaire à ce poème, que la difficulté ici consiste, non pas à résoudre d'obscures quaestiones, comme dans l'Énéide, mais à comprendre ce qui est écrit ( $a d G .1,43)$ !

103. C'est la première technique mentionnée par Sc. McGill, Virgil recomposed..., p. 11-12.

104. Ainsi, l'omission du banquet de Carthage tient peut-être au luxe excessif qui le caractérise et au fait que la nourriture y est très peu présente.

105. J'ai avancé plus haut cette hypothèse à propos de adtollitque globos au v. 2, de conclamant rapiuntque focis au v. 10.

\section{RÉSUMÉS}

L'ouvrage si stimulant de Scott Mc Gill, Virgil recomposed. The mythological and secular centos of Antiquity, Cambridge University Press, 2005, a pu susciter une attention aigue chez un nombre croissant de chercheurs pour cette forme d'écriture extraordinaire, pouvant donner lieu à des réécritures virtuoses comme celle d'Ovide via Virgile dans le centon Narcisse étudié par G. Tronchet dans le numéro 7 (2010) de de Dictynna. C'est plutôt l'ordinaire qui est au centre de cette la présente étude, où il s'agit de mieux comprendre la démarche de l'auteur du centon De panificio vis-à-vis de la poésie de Virgile. Le centon n'est pas lu ici comme une parodie, mais comme un jeu de lettré où Virgile permet peut-être, au contraire, d'élever ce qui est humble. Il témoigne en tout cas d'une très bonne connaissance de la poésie virgilienne et de certains de ses enjeux, en particulier stylistiques. L'auteur du poème $a$, en effet, réuni la plupart des passages de l'Énéide où la thématique alimentaire est en cause; il a également rapproché plusieurs passages où l'humilité intrigue, voire est problématique; enfin, il s'est peut-être donné pour but de recomposer de manière allusive et en miniature un chant particulier de l'Énéide, le chant 5 , dont chacun des principaux épisodes est représenté dans ces onze vers captivants.

\section{INDEX}

Mots-clés : centon, Virgile, réécriture, métapoétique, thèmes poétiques humbles, alimentation dans la poésie antique, pain dans l'Antiquité 